\title{
Risky Behaviors among HIV Serodiscordant and Seroconcordant Couples in Yaounde-Cameroon
}

\section{Constantin Tchakounte ${ }^{1}$, Thibau Flaurant Tchouangueu ${ }^{1,2}$, Marie Nicole Ngoufack ${ }^{2,3}$, Leaticia Grace Yatchou $^{2}$, Salomon Tchuandom Bonsi, ${ }^{1,4}$, Jean Olivier Ngono Djang ${ }^{4}$, Etienne Philemon Atabonkeng ${ }^{1}$, Jules-Rogers Kuiate ${ }^{1}$, Alexis Ndjolo ${ }^{2,5}$ and Céline Nkenfou Nguefeu ${ }^{2,6^{*}}$}

${ }^{1}$ Faculty of Science, University of Dschang, Cameroon

${ }^{2}$ Chantal Biya International Reference Centre (CBIRC), Yaounde, Cameroon

${ }^{3}$ Faculty of Sciences, University of Yaounde I, Cameroon

${ }^{4}$ Ecole des Agents Techniques Médico-Sanitaires de Yaoundé, Cameroon

${ }^{5}$ Faculty of Medicine and Biomedical sciences, University of Yaounde I, Cameroon

${ }^{6}$ Higher Teachers' Training College, University of Yaounde I, Cameroon

*Corresponding author: Céline Nguefeu Nkenfou, Associate Professor, Laboratory of Systems Biology 'Chantal Biya' International Reference Centre for Research on HIV/AIDS Prevention and Management (CBIRC), PO Box 3077, Messa, Yaoundé, Cameroon, Tel: +237-675-573-519

\begin{abstract}
Introduction: Serodiscordant HIV couples are an important source of HIV spread in sub-Saharan Africa. To limit the transmission of HIV within couples, UNAIDS has defined many strategies, including unconditional treatment of the infected partner, systematic condom use, assisted reproduction, regular screening of the negative partner. In Cameroon, there is little information on the follow up of HIV serodiscordant and seroconcordant couples. The purpose of this work was to describe risky behaviors within serodiscordant couples.
\end{abstract}

Method: This cross-sectional study was conducted in five health facilities. Self-administered questionnaires were used to collect social and demographic information, while semi-structured in-depth individual and couple interviews explored sexual relationships. Couples were then sampled and tested for HIV, HbsAg, HCV, syphilis, and chlamydia. Collected data were analyzed using the Graph Pad Prism Version 6 software.

Results: Eighteen seroconcordant and fifty tree serodiscordant couples agreed to participate in the study, where women were predominantly infected $(56.66 \%)$. Serodiscordance was known for 64 months on average. Among serodiscordant couples, $86.79 \%$ infected partners were on ARV treatment, among them 10/46 (15.22\%) stopped treatment. Sexual relationship existed in 50/53 couples, with non-systematic use of condom in 44 couples (83.01\%). Extramarital relationships were reported in 10 of the 23 serodiscordant couples $(56.60 \%)$ with libido disorders. HBsAg was most present in HIV infected women (11.32\%) while, HIV-uninfected men were most infected with viral hepatitis $C(7.55 \%)$.

Conclusion: This work has highlighted many risky behaviors in serodiscordant couples such as, interruption of ARV, non-consistence use of condoms, extra-marital relationships and co-infection with other STDs. It remains essential to provide continuous counselling to serodiscordant couples and to associate systematic STI screening in all cases of HIVI AIDS serodiscordance. In addition, stock out of ARV should be reduced through better management of procurement and supply chain of all comodities needed for the care of PLWHIV. As well the capacity of the psychosocial agent should be strengthen so as to reduce drop out of PLWHIV under treatment.

\section{Keywords}

HIVIAIDS, Risky behaviors, HIV serodiscordant couple, STIs, Yaounde
Abbreviations
HIV: Human Immunodeficiency Virus; AIDS: Acquired Im- mune Deficiency Syndrome; ART: Antiretroviral Therapy; ARVs: Antiretrovirals; STD: Sexually Transmitted Disease; STIs: Sexually Transmitted Infections; UNAIDS: Joint United Nations Programme on HIVIAIDS; UNFPA: United Nations Population Fund; CMA: Centre Médicaux d'arrondissement; 
HbsAg: Hepatitis-B surface Antigen; ELISA: Enzyme Linked Immunosorbent Assay; TPHA; Treponema Hemagglutination Assay; HCV: Hepatitis C Virus

\section{Introduction}

Management of Human Immunodeficiency Virus/ Acquired Immunodeficiency Syndrome (HIV/AIDS) pandemic remains a challenge for low-income countries where sexually transmitted disease is predominantly transmitted in stable couples in which one partner is or is not receiving care [1]. In sub-Saharan Africa, HIV serodiscordance affects one to two-thirds of couples living with HIV [2,3]. In Zambia and Rwanda, for example, new heterosexual infections in adults from serodiscordant couples range from 55.1 to $92.7 \%$ [4]. In serodiscordant couples, HIV-negative partners are at a very high risk of becoming infected [5]. HIV acquisition can be fueled by sexually transmitted infections (STIs) that cause ulceration or not. STIs greatly increase the risk of HIV infection by increasing the susceptibility of the exposed person, as well as the infectivity of an HIV-positive person [6]. The presence of an STI in an exposed person increases their susceptibility to HIV, through various mechanisms such as the presence of genital ulcers or local inflammation. For example, the presence of syphilis in an HIV infected person increases the amount of virus in the genital secretions and therefore the risk of contamination of its partners by HIV. This explains the recommendations to offer STI testing before any HIV diagnosis and vice versa [7].

Conscious of the implication of serodiscordance in the occurrence of new cases and the fueling of HIV/AIDS the pandemic, WHO through its UNAIDS program has defined strategies for HIV prevention among serodiscordant couples. These strategies include: Administration of antiretroviral therapy (ARV) unconditionally to the infected partner, using condoms consistently, using ARVs post-exposure and pre-exposure, screening the negative partner on a regular basis, as well as adequate moral support to helping couples manage HIV serodiscordance [8]. The implementation of these strategies is a struggle in Africa because of the lack of a real policy of serodiscordant couple's management according to the context. Thus, many risky behaviors (non- systematic condom use, extra-marital sex, interrupted treatment and co-infections with other STDs) have been reported in several studies including those of Ruzagira, et al. 2011 in Uganda [9], Gitonga, et al. in kenya in 2012 [10], Guira, et al. in 2012 in Burkina Faso and many other authors [11].

In Cameroon, there is no specific policy on the management of serodiscordant couples, therefore, the notion of serodiscordance in general, the national prevalence, the profile of STIs, the challenges faced by these couples, sexual behaviors within couples are poorly known and the documentation almost non-existent. De
Walque was among the authors who took an interest in serodiscordant couples in Cameroon and described sexual behaviors in this group [2].

Do they receive the appropriate support to cope with HIV serodiscordance? Do they practice their sexuality in strict compliance with HIV prevention recommendations at the individual and couple level as envisioned by WHO? The purpose of this work was to describe, 13 years after the study of Walque, et al. the risky behaviors in serodiscordant couples that may promote HIV/AIDS transmission to the seronegative partner.

\section{Materials and methods}

This was a cross-sectional study conducted from September 2016 to December 2017 in five hospitals (District Hospital of Efoulan, Nkoldongo, Biyem-Assi, cité-verte, Obala and CMA of Nkomo). Serodiscordant couples consulting in one of these hospitals were our study population, including seroconcordant couples as controls. The term couple refers to two persons living in a household and related by marriage or concubinage for a period of at least twelve months and having sex together or not. The medical records of HIV positive patients were consulted to identify serodiscordant couples, after which they were contacted by telephone. For those who consented, an appointment was made. All HIV-negative partners participating in this work repeated their test at least twice. The data were collected using a questionnaire offered to each partner separately and subsequently together. This questionnaire collected sociodemographic parameters, therapeutic follow-up, knowledge and attitudes regarding HIV/AIDS. Subsequently, blood was collected from both partners to confirm or refute their declared status.

HIV/AIDS test was carried out according to the national algorithm: A first rapid test using Determine ${ }^{\circledR}$ HIV1/2 (Abbott Diagnostic Division, Hoofddorp, The Netherlands) and a second confirmatory test with $\mathrm{KHB}^{\circledR}$ HIV (Shanghai Kehua Bio-engineering Co., Ltd., China). In case of discordant results to these two tests, the sample was re-analysed by ELISA (Avioq HIV-1 Microelisa System Rockville, USA). HBsAg, anti-HCV antibodies were investigated by the LabA$\mathrm{CON}^{\circledR}$ kit (Hangzhou Biotest Biotech Co., Ltd., China), anti-treponemal antibodies were searched using two kits, TPHA Biolabo and RPR (Biolabo SA, 02160, Maizy France) and anti-Chlamydia trachomatis antigens were investigated by the Immunocomb ${ }^{\circledR}$ Chlamydia trachomatis IgG kit (Orgenics, Israel).

\section{Statistical Analyses}

The data were entered in the excel file and analyzed using the Graph Pad Prism Version 6 software. The $\chi^{2}$ or Fischer test was used to compare the qualitative variables and the Student's test to compare the 
means with a significance level of $5 \%$.

\section{Ethical Considerations}

The study was approved by the National Research Ethics Committee for Human Health ( $N^{\circ}$ 2016/04/758/ $\mathrm{CE} / \mathrm{CNERSH} / \mathrm{SP}$ ). Before conducting the study, written informed consent was obtained and all identifying information (names, initials.) were then omitted to ensure the confidentiality. Participation was free and voluntary, participants could leave the study at any time without affecting the care they receive in these hospitals.

\section{Results}

\section{Sociodemographic characteristics of couples}

A total of 266 couples were received. Among them, those who accepted to participate were grouped as follows: 60/192 couples (31.25\%) were serodiscordant and 19/74 (25.67\%) seroconcordant. Eight couples were excluded, 7 serodiscordant couples (three for subsequent seroconversion of negative partners and 04 for insufficient data), and one seroconcordant couple (for insufficient data). All couples were heterosexual and monogamous. A proportion of $56.60 \%$ of women (30) versus $43.39 \%$ of men (23) were infected $(P=0.173)$. In addition, $60.38 \%(32)$ of the couples were married, while $39.62 \%$ (21) lived in concubinage for an average of 03 years, 03 months (ranging from one year to 17 years). Serodiscordance was known for 5 years and 2 months on average (min-max from 3 months to 15 years). All respondents reached at least primary level of education. They were more men $(8 / 53)$ with a university level than women $(5 / 53)$. The age of women ranged from 17 to 59 years with an average of 32.81; while that of the men ranged from 22 to 67 years and an average of 41.26 . A total of 37 out of $53(69.81 \%)$ couples wanted children, $18.91 \%$ (07/37) did not yet have children. Nevertheless, 9 couples (16.98\%) did not want to have children anymore (Table 1).

\section{ART observance}

We observed in this study that seroconcordant couples were more observant for their ARVs treatment

Table 1: Sociodemographic characteristics of couples.

\begin{tabular}{|c|c|c|c|c|c|}
\hline \multirow[b]{2}{*}{ Characteristics } & \multirow[b]{2}{*}{$\begin{array}{l}\text { sub- } \\
\text { characteristics }\end{array}$} & \multicolumn{2}{|c|}{$\begin{array}{l}\text { Serodiscordant couples } \\
(N=53 \text { couples })\end{array}$} & \multicolumn{2}{|c|}{$\begin{array}{l}\text { Seroconcordant couples }(N=18 \\
\text { couples) }\end{array}$} \\
\hline & & $\begin{array}{l}\text { Women } \\
\mathrm{N}(\%)\end{array}$ & $\begin{array}{l}\text { Men } \\
\mathrm{N}(\%)\end{array}$ & $\begin{array}{l}\text { Women } \\
\text { N (\%) }\end{array}$ & $\begin{array}{l}\text { Men } \\
\text { N (\%) }\end{array}$ \\
\hline \multirow[t]{3}{*}{ Educational level } & Primary & $25(47.2)$ & $25(47.2)$ & $7(38.88)$ & $3(16.66)$ \\
\hline & secondary & $25(47.2)$ & $20(37.73)$ & $9(50)$ & $11(61.11)$ \\
\hline & university & $3(5.66)$ & $8(15.09)$ & $2(11.11)$ & $4(22.22)$ \\
\hline$P$ VALUE & & \multicolumn{2}{|l|}{0.243} & \multicolumn{2}{|l|}{0.291} \\
\hline \multirow[t]{2}{*}{ Desire to procreate } & Yes & $37(69.8)$ & $37(69.8)$ & $13(72.22)$ & $13(72.22)$ \\
\hline & No & $16(30.18)$ & $16(30.18)$ & $5(27.77)$ & $5(27.77)$ \\
\hline$P$ VALUE & & \multicolumn{4}{|l|}{0.846} \\
\hline \multirow[t]{2}{*}{ HIV status } & positif & $30(56.60)$ & $23(43.39)$ & $18(100)$ & $18(100)$ \\
\hline & negative & $23(43.39)$ & $30(56.60)$ & $0(0.00)$ & $0(0.00)$ \\
\hline P VALUE & & \multicolumn{2}{|l|}{0.173} & \multicolumn{2}{|l|}{ NA } \\
\hline \multirow{2}{*}{$\begin{array}{l}\text { Alteration of sexual } \\
\text { intercourse }\end{array}$} & Yes & $30(56.60)$ & $30(56.60)$ & $9(50)$ & $9(50)$ \\
\hline & No & $23(43.39)$ & $23(43.39)$ & $9(50)$ & $9(50)$ \\
\hline P VALUE & & & 0.626 & & \\
\hline \multirow[t]{2}{*}{ Marital status } & married & $32(60.37)$ & $32(60.37)$ & $10(55.55)$ & $10(55.55)$ \\
\hline & concubinage & $21(39.62)$ & $21(39.62)$ & $08(44.44)$ & $08(44.44)$ \\
\hline P VALUE & \multicolumn{5}{|l|}{0.785} \\
\hline
\end{tabular}

$\mathrm{N}$ : Numbers, NA: Not Applicable.

Table 2: ARVs intake in serodiscordant couples and seroconcordant couples.

\begin{tabular}{|l|l|l|l|}
\hline Treatment & $\begin{array}{l}\text { Serodiscordant couples } \\
\mathbf{N}=\mathbf{5 3}\end{array}$ & $\begin{array}{l}\text { Seroconcordant couples } \\
\mathbf{N}=\mathbf{1 8}\end{array}$ & $\boldsymbol{P}$ VALUE \\
\hline Initiation of ARVs & $7(13.20 \%)$ & $0(0.00 \%)$ & \\
\hline Without interruption & $36(69.92 \%)$ & $18(100 \%)$ & 0.022 \\
\hline With interruption & $10(21.74 \%)$ & $0(0.00 \%)$ & \\
\hline
\end{tabular}

N: Numbers, ARVs: Antiretroviral. 
compared to serodiscordant couples $(P=0.022)$. All seroconcordant couples have not interrupted their treatment, while $21.74 \%$ (10/46) serodiscordant couples discontinued their treatment. These data are presented in Table 2.

\section{Sexual behaviors within couples}

Of the 53 serodiscordant couples surveyed, sexual intercourse and abstinence were observed in 50/53 (94.34\%) and 03/53 (5.66\%) couples, respectively. Altered sexual desire or maintenance of sexual desire was equitably distributed $(P=0.420)$ at $43.39 \%$ $(23 / 53)$ and $56.60 \%(30 / 53)$ between those who reported sexual desire disorders compare to those with no libido disorders in serodiscordant couples. Among couples who reported sexual desire impairment, $13.04 \%$ of couples $(03 / 23)$ opted for abstinence while sexual intercourse became increasingly rare, ranging from one to two times within three months in $17.39 \%$ of them (04/23) and one time in six months for $4.34 \%$ $(1 / 23)$ of couples. Although libido disorders were reported in $50 \%(09 / 18)$ of seroconcordant couples, the frequency of sexual intercourse was on average once per week.

Among serodiscordant and seroconcordant couples where $23 / 53$ and 09/18 couples reported having disorders of sexual desire, $43.47 \%$ (10/23) of serodiscordant couples had extramarital relationships compared to $22.22 \%(02 / 09)$ among seroconcordant couples $(P$ $=0.001$ ). Serodiscordant couples were twice at risk of having extramarital sex when libido disorders were reported than seroconcordant couples (Table 3).
The systematic use of condoms (male only) was mainly reported among seroconcordant couples (10/18) compared to serodiscordant couples $(11 / 53)$ with respective proportions of $55.55 \%$ and $20.75 \%(P=0.008)$. Similarly, the condom was more often used in seroconcordant couples $(80 \%)(8 / 10)$ than in serodiscordant couples (60.38\% (32/53)) $(P=0.008)$. Ten serodiscordant couples $(18.86 \%)$ said they had never used condom (Table 4).

\section{Prevalence of STIs (viral hepatitis B and C, syphilis, chlamydia)}

In general, none of the STIs was more present in HIV + partners than HIV - partners $(P=0.559)$ (Table 5). In HIV-infected women, $13.33 \%$ (4/30) were HBV-positive ( $\mathrm{HBsAg}$ ) versus only one among HIV-negative women $(1 / 23(4.3 \%))(P=0.039)$. HIV-positive women were 3.25 times more likely to be infected with HBV than HIV-negative women. The distribution of the other three STIs (HCV, syphilis and chlamydia) was similar between HIV + and HIV- women. In contrast, more HIV-negative men $(13.33 \%, 4 / 30)$ were infected with viral hepatitis $C(P=$ $0.039)$ compared to HIV + men $(4.3 \%, 1 / 23)$. Syphilis was more present in HIV negative men $3 / 30(10 \%)$ than HIV positif men $1 / 23(4.3 \%)$ without significant difference $(P$ $=0.40$ ). Only one case of syphilis and one of chlamydia were reported in HIV positive women and HIV negative men respectively. A case of HBV and HVC co-infection was observed in an HIV + men.

\section{Discussion}

Care for HIV serodiscordant couples requires care-

Table 3: Correlation between sexual desire disorders and extramarital relationships.

\begin{tabular}{|l|l|l|l|l|l|}
\hline & \multicolumn{2}{|l|}{ Sexual desire disorders } & & \\
\hline Extramarital relationship & Yes. N (\%) & NO. N (\%) & P VALUE & RR & OR \\
\hline Sero-discordant couples & $10(43.47)$ & $13(56.53)$ & 0.001 & 2.00 & 2.786 \\
\hline Sero-concordant couples & $2(22.22)$ & $7(77.77)$ & & \\
\hline
\end{tabular}

N: Numbers, \%: percentage, RR: Relative Risk, OR: Odds Ratio.

Table 4: Distribution of condom use among serodiscordant and seroconcordant couples.

\begin{tabular}{|l|l|l|l|}
\hline Condon usage & $\begin{array}{l}\text { Serodiscordant couples } \\
\text { N = 53 }\end{array}$ & $\begin{array}{l}\text { Seroconcordant couples } \\
\text { N = 18 }\end{array}$ & VALUE \\
\hline Never & $10(18.86 \%)$ & $0(0.00 \%)$ & 0.008 \\
\hline Sometimes & $32(60.37 \%)$ & $08(44.44 \%)$ & \\
\hline Always & $11(20.75 \%)$ & $10(55.55 \%)$ & \\
\hline
\end{tabular}

Table 5: Distribution of 04 STIs among HIV-positive partners and their HIV-negative partners in serodiscordant couples.

\begin{tabular}{|l|l|l|l|l|l|}
\hline & HbsAg & HCV & Syphilis & Chlamydia & Total \\
N (\%) & N (\%) & N (\%) & No) & N (\%) \\
\hline HIV + partner & $6(11.32 \%)$ & $1(1.88 \%)$ & $2(3.77 \%)$ & $0(00 \%)$ & $9(16.98 \%)$ \\
\hline HIV - partner & $3(5.66 \%)$ & $4(7.55 \%)$ & $3(5.66 \%)$ & $1(1.88 \%)$ & $11(20.75 \%)$ \\
\hline P VALUE (RR) & $0.459(1.57)$ & $0.100(0.25)$ & $0.757(0.66)$ & $1(0.00)$ & $0.589(0.81)$ \\
\hline
\end{tabular}

HbsAg : Hepatitis-B surface antigen, HCV : Hepatitis C virus. 
ful monitoring of both partners to limit the risk of HIV transmission as well as other STIs in couples [1]. However, the low study participation rate estimated at $68.75 \%$ and $74.33 \%$ respectively among serodiscordant and seroconcordant couples with HIV, may translate little sensitization of couples for their contribution in care in Cameroon. However, greater participation in the study would have made it possible to better appreciate the realities or experiences of HIV serodiscordant couples in Yaounde.

This study has identified some risky sexual practices, poor therapeutic compliance, and high prevalence of four STIs among serodiscordant couples compared to seroconcordant HIV couples. In serodiscordant couple, female partners were more infected with HIV than men partners but without the significant difference. This trend is observed in several studies including those of Walque, 2006, Colman-Sarfo, et al. 2017 [2,12]. This could be explained by the fact that women are physiologically more susceptible to HIV than men [13]. It should also be remembered that women can, in many cases, be forced to have sex [2].

HIV seroconcordant couples were more compliant to ARV treatment compared to serodiscordant couples where $18.86 \%$ (10) of the HIV positive partners discontinued treatment. The main reasons for discontinuation of ARVs were the lack of supplies in 4 cases (40\%) and in 6 cases $(60 \%)$ discontinuation was due to mental disorders resulting from the non-acceptance of HIV status. This risky behavior (the interruption of treatment) increases the chance of transmission to the negative partner as stopping the treatment leads to the increase of viral load, therefore the risk of transmission [14]. Previous work done by Troussier and Tourette-Jurgis in 2006 in France showed that the prevalence of sexual desire disorder was twice as high among serodiscordant couples compared with seroconcordant couples [15]. Similarly, Guira, et al. (2013) in Burkina Faso observed a very high rate of sexual desire disorders at $97.7 \%$ compared to our work where only $56.60 \%$ of couples had libido disorders. This behavior is justified mainly in all these works by the fear of infection of the negative partner [11]. The low frequency of sexual desire disorders in this work, compared to the other two studies could be justified by the increase in the number of care units and their decentralization up to the lowest level of the health pyramid that provide psychological support to the patient living with HIV [16].

Extramarital relationships (by the seronegative partner), the major consequence of sexual desire disorders in serodiscordant couples, were observed in $18.86 \%$ of couples, which represent a risk of exogenous transmission not only of HIV, but also of STIs within couples. This result is close to that found in two studies, one in New York and the other in Burkina Faso with respectively $18 \%$ and $25 \%$ of couples with extramarital relationship
$[11,17]$. It should be noted that this behavior is observed mostly in the male partner than the female partner in all these studies including the work of Walque in 2006 in 5 African countries (including Cameroon). This could be justified by the fact that in the African context, in almost all cultures, traditional customs encourage men to have multiple partners [18]. Contrary to the work of Bradley, et al. 2008 and Guira, et al. 2012 where no link was found between libido disorders and extramarital relationships, a correlation has been established in our study between extramarital relationship and sexual desire disorder [11,17]. Serodiscordant couples were twice as likely to have extramarital relationships when libido disorders were reported compared to seroconcordant couples. The frequency of $18.86 \%$ of extramarital relationships recorded in this work may not reflect the true trend of this risk behavior among couples because one of the weaknesses of this study was the fact of having interviewed 29 couples (54.72\%) together. In fact, these couples claimed to have nothing to hide and demanded to participate together in the study. All the cases of extramarital affairs reported came from the $45.28 \%$ (24/53) couples interviewed separately. These findings suggest an urgent need to strengthen couples' counseling on a psychological level in order to help them manage serodiscordance [19]. The male condom was more frequently used in seroconcordant couples compared to serodiscordant couples. This can be explained by the fact that both partners who are infected regularly receive advice on infection management, so they can easily apply HIV/AIDS prevention strategies compared to serodiscordant couples where only one partner is involved and benefits from the above mentioned advices. Studies have shown that the correct use of condoms reduces the risk of HIV transmission by $80 \%$ [20]. Condom use in a non-systematic way or never as declared by certain serodiscordant couples (79.24\%) represents a major endogenous risk for the infection of the seronegative partner. This proportion of $79.24 \%$ is higher than that of Guira, et al. [11] where $59.5 \%$ of couples did not use condoms consistently, as well as those of Colman-Sarfo, et al. [12] where $40 \%$ did not use condoms. These findings suggest the need to define specific control strategies for this group in order to limit the spread of HIV infection because even in the case of undetectable viral load, UNAIDS recommends the consistent use of condoms for HIV in serodiscordant couples [1]. The main reason for not using condom was the need to procreate in $69.81 \%$ of cases, and this behavior is observed in couples with no children as well as those with children. Moreover, in the African sociocultural context, procreation is a social pressure in the life of a couple [21]. Low medical assistance is therefore unfavorable to procreation without risk of HIV transmission, including STIs to seronegative partners in serodiscordant couples [22,23]. The distribution of STIs in the HIV positive and HIV negative partners showed that, apart 
from HbsAg which was more present in the HIV-positive partner, the rest of the three STIs were more prevalent in the HIV-negative partner, which is surprising when we know that HIV infection promotes the occurrence of other diseases in the sense that it decreases the immunity [8]. This observation may be justified by the fact that HIV-negative partners may be more susceptible to extramarital relationships, which predisposes them to exogenous exposure to STIS [24]. HIV/AIDS influences the natural history of HBV [25]. In this work, HIV-positive women were more infected with viral hepatitis $B$ than HIV-negative women with a risk estimated at 3.25 times to contract viral hepatitis B when the woman was HIV positive. This co-infection, estimated at $13.33 \%$ among women in this work, is greater than $1 \%$ found in the work of Dovonou et al., in 2015 in Benin [26]. This difference in outcome could be justified by differential distribution of viral hepatitis $B$ according to regions in sub-Saharan Africa [27]. Although HIV influences the natural history of viral hepatitis C as well [28], HIV-negative men were more infected with viral hepatitis $C$ than HIV positive men. This observation could be explained by the fact that HIV-negative men would engage in extramarital affairs and therefore expose themselves to the risk of acquisition of STDs than HIV infected with reduced libido.

\section{Conclusion}

In light of the results of this work, which revealed many risky behaviors among serodiscordant couples such as interruption of ARV taking, non-consistence use on condoms and the extra marital relationship, it remains essential to define specific HIV/AIDS prevention strategies among serodiscordant couples as a means of limiting new infections. Targeted counselling should be developed to promote assisted and safe reproductive methods in serodiscordant couples who need to procreate. With regard to STIs, it will be necessary to associate their systematic screening not only in serodiscordant partner who is invited to be tested for HIV infection on a regular basis, but also in the general population.

\section{Acknowledgements}

We thank all participants who made this work possible, as well as all the health staff who helped in the implementation of the study.

\section{Conflict of Interest}

Authors do not declare any conflict of interest.

\section{References}

1. Report on the global AIDS epidemic (2010). Joint United Nations Programme on HIVIAIDS (UNAIDS).

2. De Walque (2006) Discordant couples HIV infection among couples in Burkina Faso, Cameroon, Ghana, Kenya and Tanzania. Development Research Group The World Bank $1-28$.

3. Cohen MS, McCauley M, Gamble TR (2012) HIV treatment as prevention and HPTN 052 Curr Opin HIV AIDS 7: 99105.

4. Dunkle K, Stephenson R, Karita E, Chomba E, Kayitenkore K, et al. (2008) New Heterosexually Transmitted HIV Infections in Married or Cohabiting Couples in Urban Zambia and Rwanda: An Analysis of Survey and Clinical Data. Lancet 371: 2183-2191.

5. Allen S, Meinzen-Derr J, Kautzman M, Zulu I, Trask S, et al. (2003) Sexual behavior of HIV discordant couples after HIV counseling and testing. AIDS 17: 733-740.

6. Cohen M, Hellmann N, Levy J, DeCock K, Lange J (2008) The spread, treatment, and prevention of HIV-1: evolution of a global pandemic. J Clin Invest 118: 1244-1254.

7. La Ruche G, Goulet V, Bouyssou A, Sednaoui P, De Barbeyrac B, et al. (2013) Epidémiologie actuelle des infections sexuellement transmissibles bactériennes en France. Press Med 42: 432-439.

8. World Health Organization (2012) Guidelines on couple HIV testing and counselling including antiretroviral therapy for treatment and prevention in serodiscordant couples: Recommendations for a public health approach. Geneva, Switzerland.

9. Ruzagira E, Wandiembe S, Abaasa A, Bwanika A, Bahemuka U, et al. (2011) HIV incidence and risk factors for acquisition in HIV discordant couples in Masaka, Uganda: an HIV vaccine preparedness study. PLoS One 6: 24037.

10. Gitonga M, Ballidawa J, Ndege S (2012) Challenges and Coping Strategies Among Couples of Mixed HIV Status Presenting at a Large Comprehensive Care Center in Eldoret, Kenya. JBAH 2: 18-27.

11. Guira O, Tieno H, Sawadogo S, Drabo J (2012) Sexuality and risk for sexual transmission of HIV among serodiscordant couples in Ouagadougou, Burkina Faso. Bull Soc Pathol Exot 106: 43-47.

12. Coleman-Sarfo K, DeGraft K (2017) Challenges Faced by HIV Positive Partners Living in Discordance with HIV Negative Partners: A Case Study of 37 Military Hospital. Clinical Research in HIV/ AIDS 4: 1-11.

13. Serge-Clotaire B, Joseph F, Edson-Joan B, Georges N, Marie-Josée E, et al. (2015) Distribution épidémiologique de l'infection à $\mathrm{VIH}$ chez les femmes enceintes dans les dix régions du Cameroun et implications stratégiques pour les programmes de prévention. Pan Afr Med J 20: 79.

14. Supervie V (2013) Antiretroviral drugs-based HIV prevention methods: What impact on the HIV epidemic. Med Sci (Paris) 29: 373-382.

15. Troussier T, Tourette-Turjis $C$ (2006) The quality of life from a sexual and emotional point of view has a positive effect on prevention for patients living with HIV. Sexologies 15: $165-175$

16. Eboko F, Abé C, Laurent C (2010) Accès décentralisé au traitement du $\mathrm{VIH} / \mathrm{sida}$ : Evaluation de l'expérience camerounaise. Sciences Sociales et Sida. ANRS.

17. Bradley M, Remien R, Dolezal C (2008) Depression symptoms and sexual HIV risk behavior among serodiscordant couples. Psychosomatic Medicine 70: 186-191.

18. Women and HIVIAIDS (2004) Confronting the Crisis. UNFPA, UNAIDS, UNIFEM.

19. Julie A, Kevin R, George P, Caitlin E, Michael D (2008) HIV Voluntary Counseling and Testing and Behavioral Risk Reduction in Developing Countries: A Meta-analysis, 19902005. AIDS Behav 12: 363-373. 
20. Weller S, Davis-Beaty K (2007) Condom effectiveness in reducing heterosexual HIV transition. Review of the Cochrane Library. Cochrane Coll 4: 1-22.

21. Rispel C, Metcalf C, Moody K, Cloete A, Caswell G (2011) Sexual relations and childbearing decisions of HIV-discordant couples: an exploratory study in South Africa and Tanzania. Reprodu Health Matters 19: 184-193.

22. Ohl J, Partisani M, Wittemer C, Schmitt MP, Cranz C, et al. (2003) Assisted reproduction techniques for HIV serodiscordant couples: 18 months of experience. Human Reproduction 18: 1244-1249.

23. Ohl J, Partisani M, Wittemer C, Lang J, Viville S, et al. (2005) Encouraging results despite complexity of multidisciplinary care of HIV-infected women using assisted reproduction techniques. Human Reproduction 20: 3136-3140.

24. Bunnell R, Nassozi J, Marum E, Mubangizi J, Malamba S, et al. (2005) Living with discordance: Knowledge, challenges, and relationship strategies of HIV-serodiscordant cou- ples in Uganda. AIDS Care 17: 999-1012.

25. Amidou S, Dovonou C, Houehanou C, Kpangon A, Ahanhanzo-Glele R, et al. (2018) Impact of HIV status on the overall prevalence of chronic hepatitis $B$ infection in Parakou, Benin. Pan Afr Med J 30: 180.

26. Dovonou C, Amidou S, Kpangon A, Traore Y, Godjedo T, et al. (2015) Prevalence of hepatitis $B$ in people infected with HIV in Parakou in Benin. Pan Afr Med J 20: 125.

27. Lukhwareni A, Burnett R, Selabe S, Mzileni M, Mphahlele M (2009) Increased detection of HBV DNA in HBsAg-positive and HBsAg-negative South African HIVIAIDS patients enrolling for highly active antiretroviral therapy at a Tertiary Hospital. J Med Virol 81: 406-412.

28. Vallet-Pichard A, Pol S (2006) Natural history and predictors of severity of chronic hepatitis $\mathrm{C}$ virus (HCV) and human immunodeficiency virus (HIV) co-infection. Journal of Hepatology 44: S28-34. 